\title{
A case of intussusception caused by diffuse large B cell lymphoma in ileocecal region
}

\author{
Shuaiyang Zhou ${ }^{1 *}$ (D), Wen Zhang ${ }^{1 *}$ (1), Jianzhong $\mathrm{Wu}^{2}$ (1), Zhi Zhang ${ }^{2}$ (1), Zhenguo Qiao ${ }^{1}$ (c) \\ ${ }^{1}$ Department of Gastroenterology, Suzhou Ninth People's Hospital, Affiliated Wujiang Hospital of Nantong University, Suzhou, China \\ ${ }^{2}$ Department of General Surgery, Suzhou Ninth People's Hospital, Affiliated Wujiang Hospital of Nantong University, Suzhou, China
}

\begin{abstract}
A 28-year-old female patient was admitted to our hospital with the complaint of "intermittent blood defecation for more than ten days." Physical examination was unremarkable except for anemic appearance. Abdominal computed tomography (CT) showed a soft tissue shadow in the ileocecum along with multiple small lymph nodes in the ileocecal part (Figure 1a). Then, the patient underwent laparoscopic-assisted radical right hemicolectomy. Intraoperative investigation revealed that the lesion was located in the ileocecal region, and the terminal ileum was inserted into the ascending colon, the size of which was about $4 \times 3 \mathrm{~cm}$. There was no enlarged lymph node in the mesenteric root, so ileocecal carcinoma was considered (Figures 1. b, c). Postoperative pathology showed diffuse infiltration by atypical lymphoid cells (Figure 1d). Highly atypical tumor cells characterized by a large volume, sparse cytoplasm, irregular nuclei, and coarse chromatin could be seen under a high-power field (Figure 1e). Immunopathology: for the tumor tissue, CD10 and CD79a were positive (Figure 1f); CD20 was positive (Figure 1g); BCL-6 and Ki-67 of about $90 \%$ were positive (Figure $1 \mathrm{~h}$ ); C-MYC of about $3 \%$ was positive; and CK, CD23, CD3, MUM-1, BCL-2, CD5, CyclinD1, and Epstein-Bar virus were positive. The patient was then transferred to the hematology department for further treatment.
\end{abstract}

Intussusception is an obstructive disease caused by the insertion of a segment of the bowel into a similar bowel cavity. Most intestinal intussusceptions have obvious mechanical causes, such as intestinal polyps, intestinal tumors, intestinal duplication, and so on. As a kind of non-Hodgkin's lymphoma, diffuse large B-cell lymphoma affects the gastrointestinal tract mainly in the ileum. However, there are few reports of intussusception caused by diffuse large B-cell lymphoma in the ileocecum. Adult intussusception is rare, accounting for only about $1 \%$ of intestinal obstruction, and the small intestine is more affected than the colon. More than half of the adult intussusception is attributed to intestinal malignant tumors. Studies have found that the most common cause of ileocolic intussusception is primary adenocarcinoma, and other

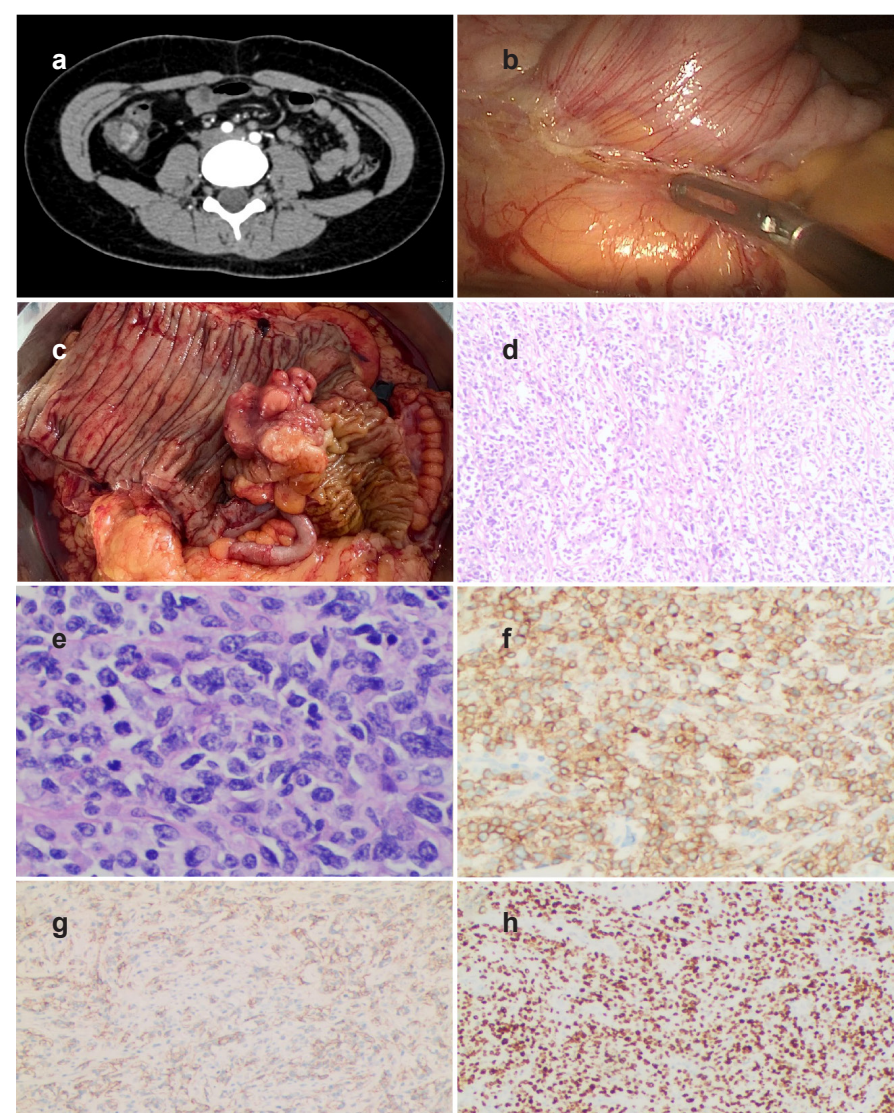

FIG.1. (a-h). Computed tomography scan of the abdomen showing that the ileocecal end is nested in the colon, with a local vortex sign (a). The lesion is located in the ileocecal region, the size of which was about 4 $\times 3 \mathrm{~cm}(\mathrm{~b}, \mathrm{c})$. Postoperative pathology showing a diffuse infiltration by lymphoid cells $(x 100)(D)$. Highly atypical tumor cells can be seen under a high-power field (x 400) (e). Positive staining for CD79a in the tumor cells (immunohistochemistry $x$ 200) $(f)$. Positive staining for CD20 in the tumor cells (immunohistochemistry $x 100)(g)$. High proliferative index with Ki-67 (immunohistochemistry x100) (h).

*Zhou $S$ and Zhang $W$ contributed equally to this work.

Address for Correspondence: Zhenguo Qiao, Department of Gastroenterology, Suzhou Ninth People's Hospital, Affiliated Wujiang Hospital of Nantong University, Suzhou, China e-mail: qiaozhenguo@ntu.edu.cn; qzg66666666@163.com

Received: April 29, 2020 Accepted: September 8, $2020 \bullet$ DOI: 10.4274/balkanmedj.galenos.2020.2020.4.223

Available at www.balkanmedicaljournal.org

ORCID iDs of the authors: S.Z. 0000-0001-9922-0561; W.Z. 0000-0003-4936-9100; J.W. 0000-0001-5767-264X; Z.Z. 0000-0003-3777-108X; Z.Q. 0000-0002-9079-956X.

Cite this article as:

Zhou S, Zhang W, Wu J, Zhang Z, Qiao Z. A case of intussusception caused by diffuse large B cell lymphoma in ileocecal region. Balkan Med J. 2021;38(2):133-134.

Copyright@Author(s) - Available online at http://balkanmedicaljournal.org/ 
rare causes, such as lymphoma, are rarely reported..$^{1-3} \mathrm{CT}$ is the first choice to diagnose the disease. A variety of CT findings, such as typical concentric circle sign, kidney shape, double tube sign, and so on, are helpful for our diagnosis. Once intussusception is diagnosed, intervention should be carried out as soon as possible to avoid intestinal necrosis. Adult intussusception often starts more rapidly and is often secondary to tumors. Surgical treatment is usually recommended when the diagnosis is clear.

Diffuse large B-cell lymphoma is a common type of non-Hodgkin's lymphoma, accounting for almost one third of all cases. More than $50 \%$ of patients have extranodal lesions when diagnosed. The most common extranodal lesions occur in the gastrointestinal tract and bone marrow. This time, the first symptom of our patient was intestinal symptoms. In addition to the surgical treatment of intussusception, treatment of the primary disease cannot be ignored. ${ }^{4}$ The treatment of diffuse large B-cell lymphoma has gradually entered the mode of targeted drug combined chemotherapy. However, we need to continuously adjust the corresponding treatment plan according to the specific condition to improve the survival rate.

Patient Consent for Publication: Written consent was obtained from the patient for publication of the data in this study.

Author Contributions: Concept - Z.Q.; Supervision - Z.Q.; Data Collection and/or Processing - J.W., Z.Z., S.Z.; Analysis and/or Interpretation - W.Z.; Literature Search - W.Z.; Writing - S.Z.

Conflict of Interest: The authors have no conflicts of interest to declare.

Funding: The authors declared that this study has received no financial support.

\section{REFERENCES}

1. Eisen LK, Cunningham JD, Aufses AH. Intussusception in adults:institutional review. J Am Coll Surg. 1999;188:390-395. [Crossref]

2. Hong KD, Kim J, Ji W, Wexner SD. Adult intussusception: a systematic review and meta-analysis. Tech Coloproctol. 2019;23(4):315-324. [Crossref]

3. Azar T, Berger DL. Adult intussusception. Ann Surg. 1997;226(2):134-138. [Crossref]

4. Hunt KE, Reichard KK. Diffuse large B-cell lymphoma. Arch Pathol Lab Med. 2008;132(1):118-24. [Crossref] 